\title{
Kikuchi-Fujimoto Disease Associated to the Epstein-Barr Virus. A Type of Rare Necrotizing Lymphadenitis and Its Differential Diagnosis
}

\author{
Mónica Belinda Romero Guadarrama ${ }^{1,2 *}$, Oscar Daniel Guzmán-Aguilar ${ }^{1,2}$, \\ Adriana Carolina López-Ugalde ${ }^{3}$, José Samuel Almeida Navarro ${ }^{1,2}$, Humberto Cruz-Ortíz ${ }^{1,2}$ \\ ${ }^{1}$ Unidad de Patología, Hospital General de México “Dr. Eduardo Liceaga”, México D.F., México; ${ }^{2}$ Facultad de Medicina, \\ Universidad Nacional Autónoma de México, México D.F., México; ${ }^{3}$ Unidad de Otorrinolaringología, Hospital General de México \\ “Dr. Eduardo Liceaga”, México D.F., México. \\ Email: *monicaromero@att.net.mx, *monica62romero@gmail.com
}

Received August $17^{\text {th }}, 2013$; revised September 20 $0^{\text {th }}, 2013$; accepted September $28^{\text {th }}, 2013$

Copyright (C 2013 Mónica Belinda Romero Guadarrama et al. This is an open access article distributed under the Creative Commons Attribution License, which permits unrestricted use, distribution, and reproduction in any medium, provided the original work is properly cited.

\begin{abstract}
Introduction: Kikuchi-Fujimoto disease (KFD), also known as histiocytic necrotizing lymphadenitis, is a specific and self-limited disease; its etiology is unknown. Some causal microorganisms have been proposed. The objective of the present article is to emphasize the clinicopathological characteristics of this disease that has been associated to the Epstein-Barr virus and to compare the histological changes with other types of necrotizing lymphadenopathies. Material and Methods: We studied 32 patients of the Surgical Pathology Service with necrotizing lymphadenitis, diagnosed in the years from 2004 to 2012 to found more cases of this rare disease in our Institution. Patients were 18 women and 14 men with an average age of 37 years. Results: The lymph nodes were cervical and axillary ones, some were associated to autoimmune diseases and no cause was identified in others. One of the cases, was diagnosed as KFD, presented morphological changes characteristic of this disease, such as subcapsular lymphoid follicles, zones with cell debris, epithelioid macrophages, clear-cytoplasm histiocytes, and immunoblast-reactive lymphocytes. Immunohistochemical markers were determined, such as CD20, CD2, CD4, CD8, CD68, lysozyme, CD56, granzyme B and EBER, which demonstrated the presence of $\mathrm{B}$, T lymphocytes, histiocytes and cells positive to EBER. Histological changes in KFD occurred in three stages: proliferative stage, necrotizing, and xanthomatous. It is important to identify the histological stages of the disease because a differential diagnosis must be performed in regard to lymphadenopathies with necrosis and diverse types of lymphomas. Conclusion: We present a case of necrotizing lymphadenitis (KFD) associated to the Epstein-Barr virus and in some cases it is not possible to render a specific diagnosis based on morphologic findings, alone, and a diagnosis of necrotizing lymphadenitis may be used.
\end{abstract}

Keywords: Kikuchi-Fujimoto Disease; Epstein Barr Virus

\section{Background}

Causes of lymphadenopathy must always be identified in each patient. The histopathological profile allows knowing in most cases the cause of such growth, which can be diagnosed to a primary or secondary (metastasis) neoplasm, or some types of hyperplasia or inflammatory processes, which are important to recognize, since the pathologist must identify the changes associated to necrosis to be able to distinguish reactive processes of any malignant growth [1-3].

\footnotetext{
"Corresponding author.
}

Diverse reactive symptoms can be associated to necrosis and polymorphous infiltrates. These can be observed in viral infections, such as those caused by the EpsteinBarr virus (EBV), in infectious mononucleosis where the infiltrate is diffuse, polymorphous, with apoptotic debris, and zonal necrosis. In the lymphadenitis due to Herpes simplex virus and in dermatopathic lymphadenitis predominating a T-cell hyperplasia associated with lymph node that drains from irritated, infected or inflamed skin sites, something similar occurs with the necrotizing lymphadenitis associated to systemic lupus erythematosus. Other types of lymphadenitis of unknown etiology, 
where a viral origin is assumed, are Kawasaki disease and Kikuchi-Fujimoto disease (histiocytic necrotizing lymphadenitis). The latter was described separately, almost simultaneously [4,5], in Japan by Kikuchi and Fujimoto in young oriental females and considered as a benign self-limited disease, characterized by cervical lymphadenopathy and systemic symptoms. Its etiology is still unknown, although several microorganisms, including viruses and bacteria, have been proposed as causes of the disease. The objective of the present study is to report a case of KFD in a 16-year-old female adolescent, with cervical lymphadenopathy with extensive necrosis, systemic manifestations, and transien affection of the skin. Biopsy of cervical lymph node revealed zonal necrosis, abundant cell debris, histiocytes, and a variable number of mature lymphocytes. Immunohistochemistry tests revealed polytypical proliferation of $\mathrm{B}$ lymphocytes, $\mathrm{T}$ lymphocytes, T/cytotoxic lymphocytes, and the presence of the EBV (Epstein-Barr-Virus) as demonstrated by in situ hybridization. This disease is extremely rare in our institution and the diagnosis must include such infarcts that may be associated with lymphoma, Infectious lymphadenitis caused by mycobacterium, HIV (Human Immunodeficiency Virus) and others rare disease; we discuss its differential diagnosis from reactive lymphadenopaties and lymphomas with necrosis, because the treatment of the patients with different types of lymphadenopaties is different.

\section{Material and Methods}

The electronic database of the Surgical Pathology Service of the Pathology Unit of the General Hospital of Mexico was retrospectively reviewed. We selected those cases with the diagnosis of "necrotizing lymphadenitis", without any other specification, that occurred in a 8-year period (2004 to 2012). We also reviewed the histological sections stained with hematoxylin-eosin and with special stains such as Shiff's periodic stain, Groccot and ZielNelsen stains. We gathered the clinical data from the available files and eliminated those cases with diagnosis of necrosis by tuberculosis or another infectious agent. From the patient with KFD diagnosis, we obtained the clinical information, radiological images, laboratory data, description of cutaneous injuries, excisional biopsy, and reviewed the immunostaining was performed using the (avidin-biotin peroxidase method) accord to the manufacturer's instructions (DAKO Cytomation, Ely, United Kingdom) and the following markers were used: CD20, CD3, CD4, CD8, CD56, CD68, Lisozyme and Granzyme B.

We also performed in situ hybridization for the RNA of the EBV. For the EBER probe, we used the fluorescein-labeled peptide to detect the nuclear presence of coded RNA. Conjugated alkaline phosphatase, rabbit antibody, and isothiocyanate-fluorescein were added, followed by the substrate nitro-blue tetrazolium and 5bromo-4-chloro-3'-indolyphosphate (NBT/BCIP; Roche Diagnostics, Indianapolis, IN, USA). Contrast was achieved with Hill's hematoxylin and the final procedure was performed as in the described immunohistochemical technique. A nasal-type T/NK lymphoma was used as positive external control for the EBV.

\section{Results}

We studied 32 patients with the diagnosis of necrotizing lymphadenitis in the 8-year period (2004-2012), 18 were women (56.2\%) and 14 (43.7\%) were men. The youngest patient was a 6-year-old girl and the oldest was a 74year-old woman. Average age was 38.7 years. Twenty four cases (75\%) corresponded to the cervical region, four cases $(12.5 \%)$ to the axillary region, three cases $(9.3 \%)$ were inguinal, and $1(3.1 \%)$ corresponded to the retroperitoneum. In three patients, it was possible to confirm the association of necrotizing lymphadenitis with autoimmune diseases such as lupus erythematosus (2 cases), rheumathoid arthritis (1 case) (Table 1), only in a 16year-old adolescent was the KFD associated to the EBV; clinically, she presented increased size of the cervical lymph node of 2-month evolution, accompanied by asthenia, adynamia, fever, athralgia, and vesicle-shaped cutaneous injuries in the fingers. Laboratory tests such as blood tests and the search for antinuclear antibodies were normal or negative. The CAT scan (computerized axial tomography) of the neck revealed malignant-suggestive jugular lymphadenopathy of more than $3 \mathrm{~cm}$ (Figure 1). In the remainder 28 patients (87.5\%) no associated disease could be documented.

Biopsies of patients with associated autoimmune disease revealed the presence of lymphoid cells in diverse maturation stages, predominating small lymphocytes, histiocytes, and necrosis. In the two cases associated to systemic lupus erythematosus, hematoxylin bodies were found in the paracortex zone; in the patient with rheumatoid arthritis the biopsy revealed periarterial and periarteriolar fibrosis with zonal necrosis in most of the histological section, with lymphoid infiltrate, occasional plasmatic cells, histiocytes, abundant cell debris and lack of hematoxilin bodies. Large areas of zonal necrosis, cell debris, scarce lymphocytes in diverse maturation stages and histiocytes, and lack of polymorphonuclear cells (Figures 2 and 3) were observed in the patient with KFD. In the cases of reactive and unspecific necrotizing lymphadenitis, marked necrosis was observed in the cortical and paracortical areas together with lymphocytes in diverse maturation states predominating mature lymphocytes, as well as abundant cell debris, scarce plasmatic 
Table 1. Clinical changes in 32 patients with necrotizing lymphadenitis.

\begin{tabular}{cccc}
\hline Sex & Age & Localization & Clinical findings \\
\hline Female (18 cases) & $6-74$ yo & $\begin{array}{c}\text { Cervical (14) Axilar (3) } \\
\text { Inguinale (1) } \\
\text { Cervical (10) Axilar (1) }\end{array}$ & $\begin{array}{c}\text { LES (2) Enlargement of the Lymph node affected } \\
\text { (18cases) and pain (7cases) }\end{array}$ \\
Male (14 cases) & $16-64$ yo & $\begin{array}{c}\text { RA (1) Enlargement of the Lymph node affected } \\
\text { (14 cases) and pain (3) }\end{array}$ \\
\hline
\end{tabular}

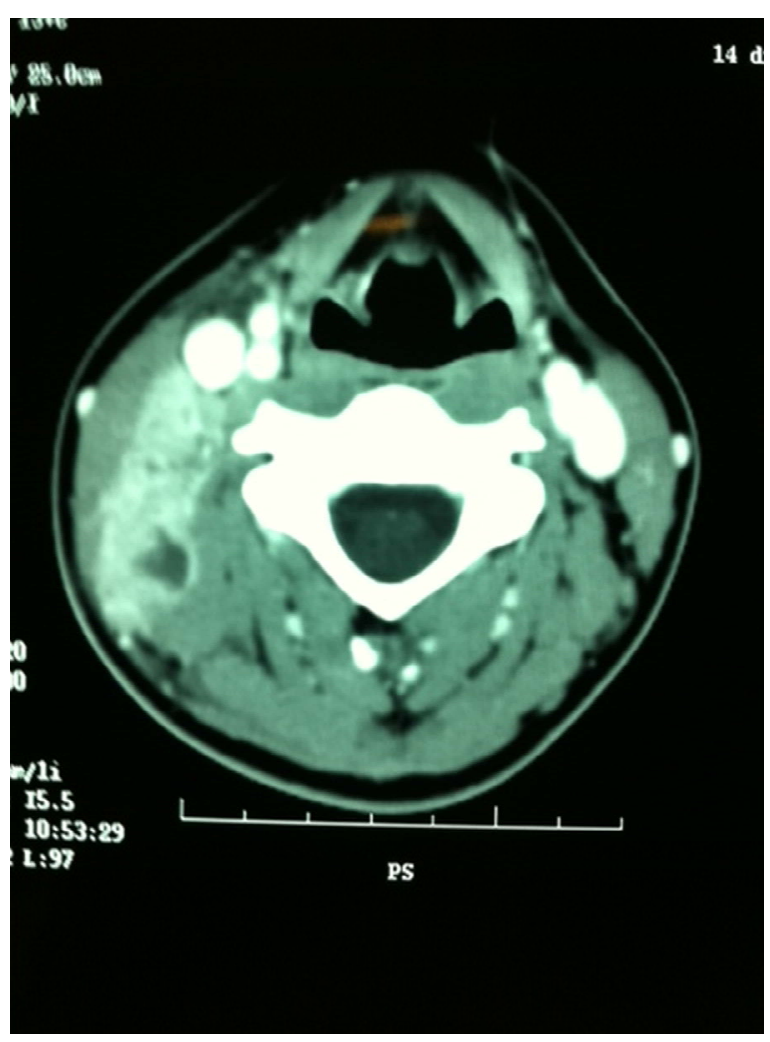

Figure 1. Axial computarized tomography of cervical Lymph node where shown enlargement of the lymph nodes with necrosis.

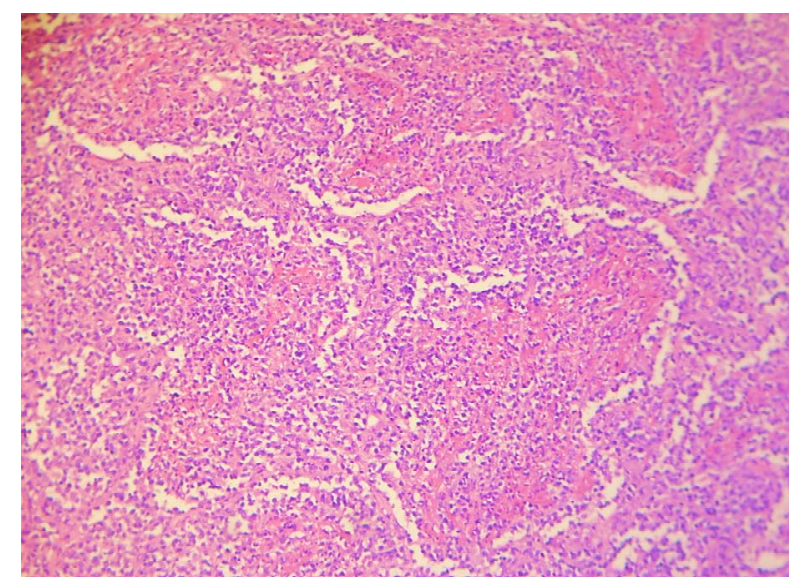

Figure 2. The biopsy in a young female, where we can see few necrosis, lymphocytes and histiocytes $(10 \times$ Hematoxilineosin stain).

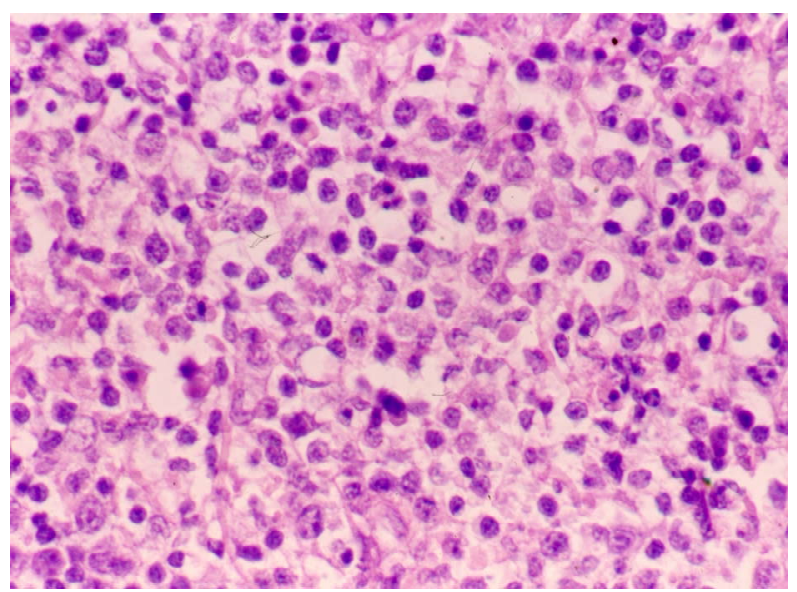

Figure 3. A high magnification we can see the histiocytes and the immunoblastic cells $(40 \times$ H-E stain).

cells and histiocytes, lack of hematoxylin bodies, periarteriolar fibrosis, squamous histiocytes, and polymorphonuclear infiltrates.

Special stains in search of microorganisms were negative and immunohistochemical reactions demonstrated scarce B cells, T cells, and some lysozyme-positive histiocytes.

In the Kikuchi-Fujimoto disease case, the presence of scarce B cells and T CD 2-positive cells was documented, and the relation of T CD 8-positive lymphocytes with CD 4 was higher in the first.

Expression of T cytotoxic cells positive to Granzyme B was observed, as well as scarce CD 56 positive cells, scarce B cells, and nuclear expression to EBER in many lymphoid cells (Figure 4).

\section{Discussion}

Necrotizing lymphadenitis is unusual and can be associated to a large variety of systemic and infectious diseases. Among the former, autoimmune diseases like systemic lupus erythematosus (SLE) must be considered is characterized by irregularly shaped, focal or extensive, necrosis sometimes accompanied by LE bodies or hematoxylin bodies that are heavily hematoxylin-stained homogeneous nuclear remnant of cells reacted with ANAs (Anti-nuclear antibodies) and are strongly indicative of SLE (Figure 5). Inmunohistochemistry would demonstrate the lymphoid follicles are mixture of small and 


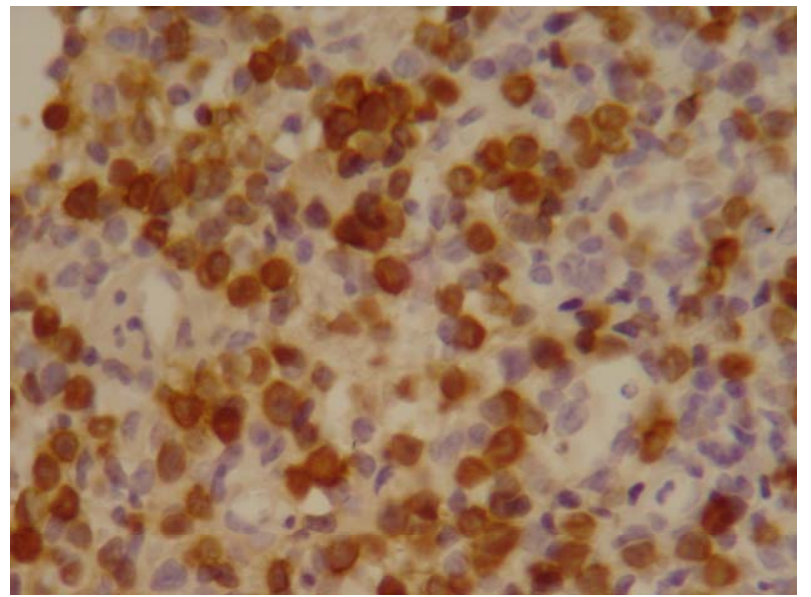

Figure 4. Eber is positive in lymphoid cells in the young patient with KFD (40× in situ hibridization).

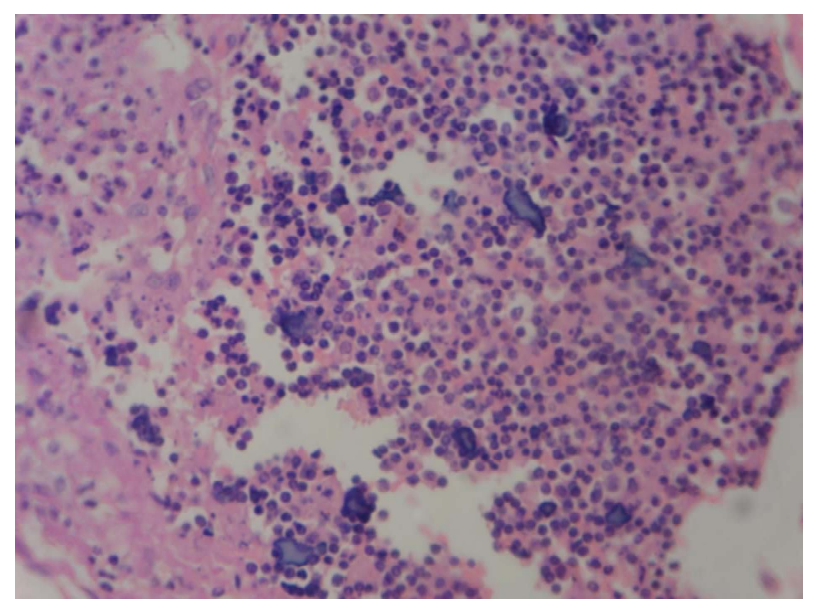

Figure 5. Necrotizing lymphadenitis by Erithematosus Lupus, is evident the hemathoxifilic bodies (15 $\times$ H-E stain).

medium-size lymphocytes and immunoblasts that are positive for B-cell or T-cell markers CD4 and CD8 stains would show a mixed nature of the interfollicular T lymphocytes. In many occasions it is not possible to document the cause or causes of lymphadenitis associated with necrosis, therefore, clinical information and laboratory tests are needed to identify the probable etiology, and the different morphological patterns of lymph node must always be taken into account, as these will vary depending on the etiological agent; the most common lymph node growth corresponds to hyperplasias, of which the following varieties are known: follicular hyperplasia, hyperplasia of marginal B zone, paracortical lymphoid hyperplasia, sinusal hyperplasia and their combination, although in these hyperplasia patterns, it is not common to find zones of necrosis [1].

In the case of acute lymphadenitis, it is common to find some type of infectious agent and the clinical symptoms are very suggestive of an infectious process associ- ated to the increase in lymph node volume [2,3]. KFD, the cause is unknown until now, infectious and autoimmune causes have been suggested, viral infections like, EBV has been shown by some authors to be linked to the disease, but the studies are not conclusive, another association is with the hemophagocytic syndrome [4,5].

Most authors agree that KFD patients present leukopenia, elevation of transaminases, dehydrogenase lactate and erythrocyte sedimentation [6,7]. In our patient, no alterations were observed in the laboratory tests. Before the lymphadenopathy, the patient presented cutaneous alterations characterized by vesicles in the fingers of both hands, which disappeared spontaneously, these cutaneous manifestations have already been documented. Reck et al. [8] reported in their study that dermatological alterations occur in $27 \%$ of patients. KFD is more common in Asiatic individuals than in the remainder world population. The ultrastructural histological changes and those revealed by histochemistry imply a hyperimmune reaction mediated by $\mathrm{T}$ cells in patients susceptible to a variety of non-specific stimuli. Some HLA class II (major histocompatibility complex) genes have been demonstrated in patients with the disease [9]. In Mexico, the disease is rare, the first two cases, in two women were reported in our institution in 1987 [10]. In 2006, Gutiérrez-Castro, et al. [11] reported a series of 14 cases, 11 women and 3 men, median age was of 37 years. The necrotizing type was the predominant histological pattern that predominated in these patients, and no association with the EBV was reported. Other cases have been reported as isolated cases in diverse national medical journals, even in pediatric age patients and in adolescents [12-14].

Diagnosis must be performed in correlation with clinical, morphological, and Immunohistochemistry data; CD 68 and lysozyme are the markers that confirm the presence of macrophages in lymphoid tissue [10]. Imaging studies such as CAT (Computarized Axial Tomography) and MRN (Magnetic Resonance Nuclear) do not help to differentiate this disease from other diseases involving lymph node, such as lymphoma, metastases, or tuberculosis; hence, excisional biopsy of affected lymph node must be done, particularly when necrotic areas are evidenced by imaging studies. In the excisional biopsy it is necessary to discard diseases that course with extensive necrosis such as hyporeactive and non-reactive tuberculosis, where poorly defined granulomas, rare giant cells, caseous necrosis and karyorrhexis are identified [15]. The presence of tuberculosis bacilli demonstrated with special stains confirm this disease, and KFD is discarded, as done in this report.

Necrotizing lymphadenitis is characterized by presenting necrotic areas with a cartographic aspect, as well as reactive lymphoid cells of immature aspect (immunoblasts), numerous histiocytes, and absence of polymor- 
phonuclear cells. Other lymphadenopathies, in some cases, can resemble slightly KFD, such as cat scratch disease, tularemia, venereal lymphogranuloma, other bacterial or even viral infections; in some cases it is not possible to establish a definite KFD diagnosis based only on the morphological changes, and the diagnosis of necrotizing lymphadenitis can be used when a characteristic pattern is shown with lack of polymor phonuclear cells [16].

It is important to keep in mind the different histological aspects of KFD, particularly for the necrotizing stage, when cells of the proliferative stage are observed [17-19] and can be confused with malignant lymphoid cells, hence, the most important differential diagnosis is with respect to lymphomas especially if abundant immunoblasts and necrosis are observed. Within this group, we have to consider B diffuse lymphomas of large cells
(Figure 6), if faced with the suspicion of this disease, other malignant cells must be searched for such as centroblasts, immunohistochemical reactions like CD20, CD4, CD8, CD68, lysozyme, and Ki 67 can help to establish the correct diagnosis [11,18-20].

The $\mathrm{T}$ lymphomas are rare, it is important to consider always the primary nasal-type T/NK lymphoma of lymph node, in this lymphoma it is possible to observe angiocentricity of neoplastic cells, marked necrosis, and pleomorphism of neoplastic cells; immunohistochemical reactions are very useful [21] (Figure 7). Other rare types of neoplasms that have to be included in the differential diagnosis are the T cells plasmacytoid leukemia, a clinicopathological entity recently described in older men. If the histiocytes of the KFD resemble sealed ring cells, scarcely differentiated metastatic adenocarcinomas must be included [11,18] (Table 2).

Table 2. Differential diagnosis of Kikuchi-Fujimoto lymphadenitis.

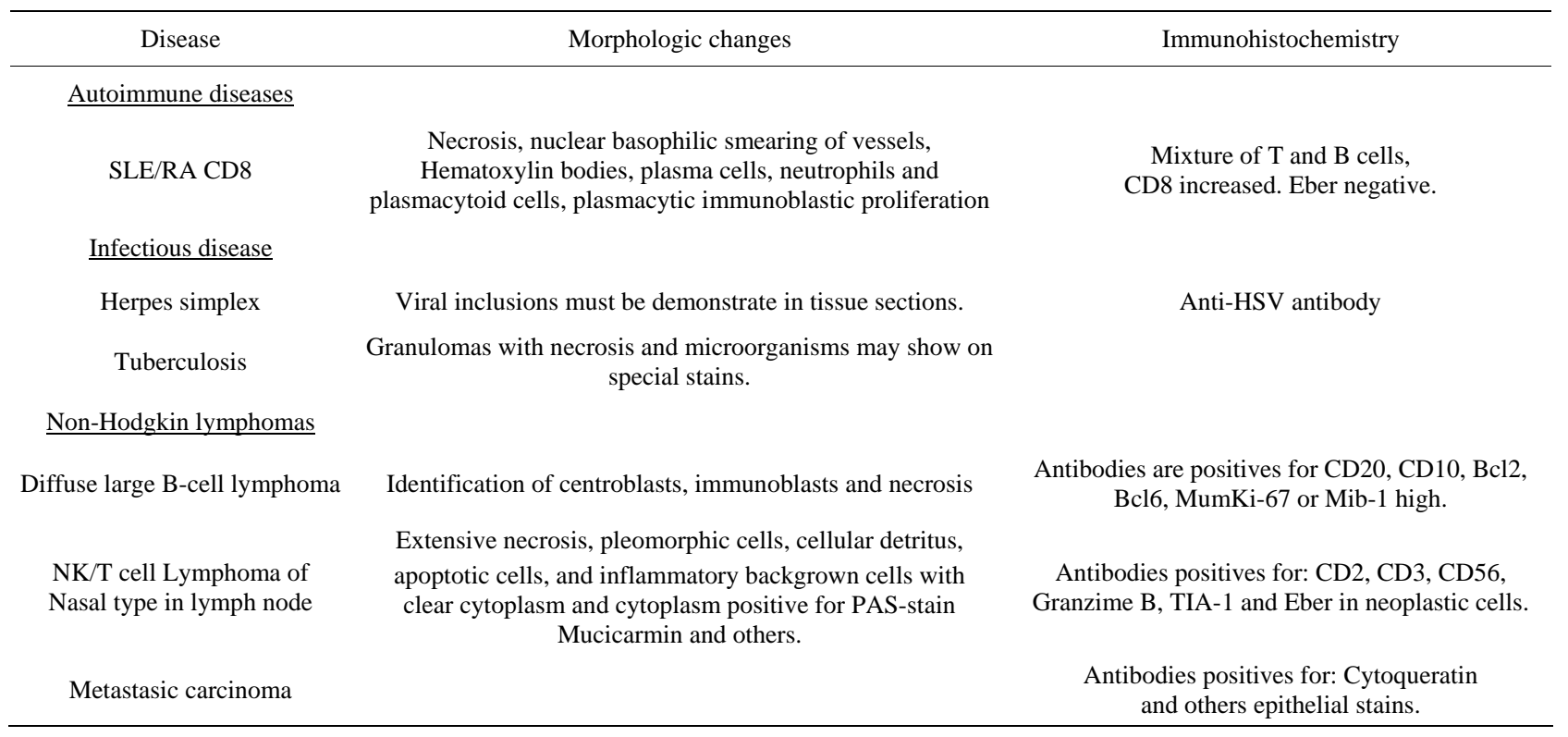

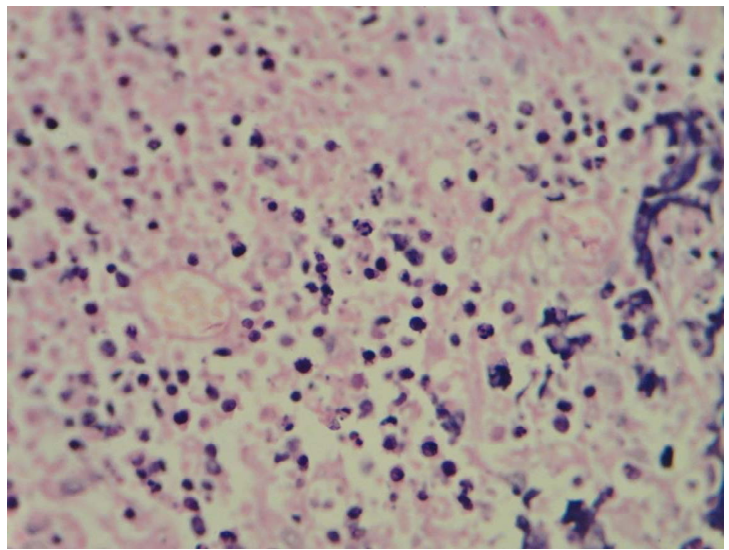

Figure 6. Large B cell with necrosis (15×, H-E stain).

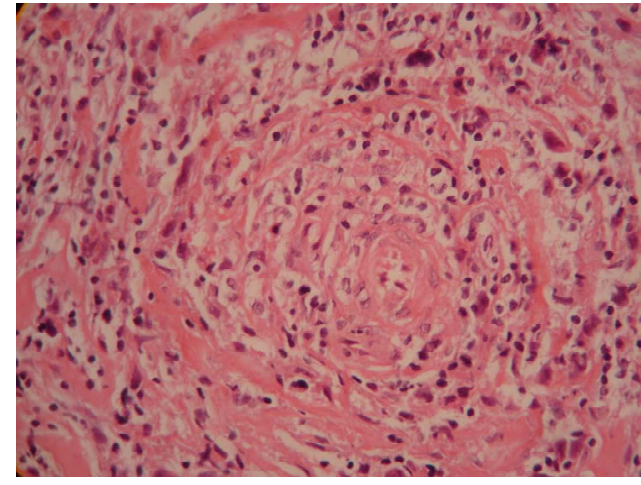

Figure 7. Lymphoma T/NK-Nasal Type of the lymph node, where we can see angioinvasion for pleomorphic tumor cells (40×, H-E stain). 


\section{Conclusion}

In conclusion, we present a rare case of KFD, in a 16-year-old adolescent, with unspecific cutaneous manifestations and cervical lymphadenopathy, with KFDcharacteristic changes, where the presence of EBV was evidenced by in situ hybridization and she's healthy two years after the diagnosis. This is the third case reported in our institution and all the previously cases diagnosed as necrotizing lymphadenitis were excluded due to the presence of polymorphonuclear leukocytes. It is important to know this rare disease in Mexico, because it is generally confused with highly malignant lymphomas or infectious processes. Necrotizing lymphadenitis is a rare finding and in some cases it is not possible to render a specific diagnosis based on morphologic findings alone and a diagnosis of necrotizing lymphadenitis alone or unspecific may be used.

\section{REFERENCES}

[1] P. Van der Valk and C. J. Meijer, "The Histology of Reactive Lymph Nodes," American Journal of Surgical Pathology, Vol. 11, No. 11, 1987, pp. 866-882. http://dx.doi.org/10.1097/00000478-198711000-00005

[2] I. L. Weissman, R. Warnke, E. C. Butcher, R. Rouse and R. Levy, "The Lymphoid System. Its Normal Architecture and the Potential for Understanding the System through the Study of Lymphoproliferative Diseases," Human Pathology, Vol. 9, No. 1, 1978, pp. 25-45. http://dx.doi.org/10.1016/S0046-8177(78)80005-7

[3] H. Stein, A. Bonk, G. Tolksdorf, K. Lennert, H. Rodt and J. Gerdes, "Immunohistologic Analysis of the Organization of Normal Lymphoid Tissue and Non-Hodgkin's Lymphomas," Journal of Histochemistry \& Cytochemistry, Vol. 28, No. 8, 1980, pp. 746-760. http://dx.doi.org/10.1177/28.8.7003001

[4] S. D. Hudnall, "Kikuchi-Fujimoto Disease: Is EpsteinBarr Virus the Culprit?” American Journal of Clinical Pathology, Vol. 113, 2000, pp. 761-764. http://dx.doi.org/10.1309/N4E2-78V9-QTFH-X4FF

[5] H. Y. Lee, Y. C. Huang, T. Y. Lin, J. C. Huang, C. P. Yang, T. Hsueh and cols, "Primary Epstein-Barr Virus Infection Associated with Kikuchi's Disease and Hemophagocytic Lympohistiocytosis: A Case Report and Review of the Literature," Journal of Microbiology, Immunology and Infection, Vol. 43, No. 3, 2010, pp. 253-257. http://dx.doi.org/10.1016/S1684-1182(10)60040-0

[6] Y. Fujimoto, Y. Kuzima and K. Yamaguchi, "Cervical Subacute Necrotizing Lymphadenitis: A New Clinicopathologic Entity,” Naika, Vol. 20, 1972, pp. 920-927.

[7] M. Kikuchi, "Lymphadenitis Showing Focal Reticulum Cell Hyperplasia with Nuclear Debris and Phagocytosis," Nippon Ketsueki Gakkai Zasshi, Vol. 35, 1972, pp. 379380.

[8] A. Reck, et al., "Kikuchi’s Disease: Case Report and Systematic Review of Cutaneous and Histopathologic Pres- entations," Journal of the American Academy of Dermatology, Vol. 59, No. 1, 2008, pp. 130-136. http://dx.doi.org/10.1016/j.jaad.2008.03.012

[9] T. Tanaka, M. Ohmori, S. Yasunaga, et al., "DNA Typing of HLA Class II Genes (HLA-DR, -DQ and -DP) in Japanese Patients with Histiocytc Necrotizing Lymphadenitis (Kikuchi Disease)," Tissue Antigens, Vol. 54, No. 3, 1999, pp. 246-253. http://dx.doi.org/10.1034/j.1399-0039.1999.540305.x

[10] M. E. Marín, H. Cruz Ortíz, B. Lauffer Dorotinsky and C. Alor, "Linfadenitis Necrosante," Revista de la Facultad de Medicina. UNAM, Vol. 30, No. 6, 1987, pp. 169-174.

[11] M. Gutiérrez-Castro, B. De León Bojorge, T. CuestaMejías, J. Baquera-Heredía, A. Padilla-Rodríguez and C. Ortíz Hidalgo, "Enfermedad de Kikuchi-Fujimoto (Linfadenitis Histiocítica Necrosante). Estudio Clinicopatológico e Inmunohistoquímico de 14 Casos y su Diagnóstico Diferencial con Otras Linfadenitis Necrozante Reactivas y Neoplásicas,” Revista de Investigación Clínica, Vol. 58, No. 5, 2006, pp. 441-449.

[12] M. Cadena Morales, G. Nuñez Zurita, N. Pérez Blanco and M. Ladrón de Guevara Méndez, "Enfermedad de Kikuchi-Fujimoto: Comunicación de un Caso y Revisión de la Bibliografía,” Anales de Otorrinolaringología de México, Vol. 53, No. 1, 2008, pp. 35-40.

[13] H. Jaramillo Ramírez, A. Morales Miguel and M. E. Marín Fregoso, "Enfermedad de Kikuchi-Fujimoto. Reporte de un Caso y Revisión de la Bibliografía,” Medicina Interna de México, Vol. 27, No. 4, 2011, pp. 403-405.

[14] P. C. Pallares-Trujillo, L. Hernández-Delgado, I. EstradaMoscoso, G. Flores-Nava and A. Lavalle-Villalobos, "Linfadenitis Histiocítica Necrosante (Enfermedad de Kikuchi-Fujimoto)," Boletín Médico del Hospital Infantil de México, Vol. 62, No. 2, 2005, pp. 136-140.

[15] G. F. Rosales-Magallanes, E. Vázquez del Río, R. Sánchez-Cisneros and J. P. Sandoval García, "Enfermedad de Kikuchi-Fujimoto en una Adolescente," Revista Mexicana de Pediatría, Vol. 79, No. 3, 2012, pp. 129-132.

[16] V. D. Ramanathan, M. S. Jawahar, C. N. Paramasivan, K. Rajarama, et al., "A Histological Spectrum of Host Responses in Tuberculous Lymphadenitis,” Indian Journal of Medical Research, Vol. 109, 1999, pp. 214-218.

[17] D. P. O’Malley, I. G. Tracy, A. Orazy and L. S. Abbondazo, "Benign and Reactive Conditions of Lymph Node and Spleen,” Atlas of Non Tumor Pathology, ARP Press, Vol. 128, 2009, p. 29.

[18] X. Bosh, A. Guilabert, R. Miguel and E. Campo, “Enigmatic Kikuchi-Fujimoto Disease a Comprehensive Review,” American Journal of Clinical Pathology, Vol. 122, 2004, pp. 141-152. http://dx.doi.org/10.1309/YF081L4TKYWVYVPQ

[19] T. Kuo, “Kikuchi’s Disease (Histiocytic Necrotizing Lymphadenitis). A Clinicopathologic Study of 79 Cases with an Analysis of Histologic Subtypes, Immunohistology and DNA Ploidy," American Journal of Surgical Pathology, Vol. 19, No. 7, 1995, pp. 798-809. http://dx.doi.org/10.1097/00000478-199507000-00008

[20] L. P. Menasce, S. S. Bannerjee, D. Edmondson, et al., 
"Histiocytic Necrotizing Lymphadenitis (Kikuchi-Fujimoto Disease): Continuing Diagnostic Difficulties," Histopathol, Vol. 33, No. 3, 1998, pp. 248-254.

[21] M. B. Romero-Guadarrama, M. A. Durán-Padilla, A. Alcántara Vásquez, M. Hernández González, K. Hopp García and S. Rivas Vera, "Linfomas no Hodgkin T Pri- marios de Ganglio Linfático. Análisis Morfológico, de Inmunofenotipo y su Asociación con Virus de EpsteinBarr en Pacientes del Hospital General de México," Revista Médica del Hospital General de México, Vol. 70, No. 4, 2007, pp. 168-174. 